\title{
A Case of Dural Arteriovenous Fistula Case in the Falx with Prominent Falcine Venous Plexus
}

Daisuke Satoh, ${ }^{1}$ Tetsuo Sasaki, ${ }^{2}$ Takehiro Yako, ${ }^{1}$ Kazuo Kitazawa, ${ }^{1}$ and Shigeaki Kobayashi ${ }^{1}$

Objective: The falx cerebri is known to have venous plexuses. Although some cases of dural arteriovenous fistula (DAVF) associated with falcine sinus have been reported, DAVF in the falx with prominent falcine venous plexus has not previously been reported.

Case Presentation: A 59-year-old male was hospitalized with head trauma. MRI incidentally showed a possible occipital DAVF. CTA and DSA revealed a DAVF in the flax with prominent falcine venous plexus. We performed a selective transarterial embolization with glue and particle, obtaining a complete occlusion of the fistula.

Conclusion: We report a rare case of DAVF in the flax with prominent falcine venous plexus that was successfully treated by a transarterial embolization.

Keywords $>$ falcine venous plexus, dural arteriovenous fistula, transarterial embolization

\section{Introduction}

The falx cerebri is an intracranial midline partition and this structure is known to have venous plexuses, mainly concentrated in its posterior one-third and inferior two-thirds in the cadaveric study. ${ }^{1)}$ On the other hand, the falcine sinus in the falx cerebri is an embryological transit venous sinus between the superior sagittal sinus (SSS) and inferior sagittal sinus (ISS) or the straight sinus. It persists $2.1 \%$ in CT examinations of adult patients. ${ }^{2)}$ Dural arteriovenous fistulas (DAVFs) can occur in the falx cerebri, and they may involve either falcine sinus or falcine venous plexus. Some authors have reported the DAVFs in the falx cerebri as the falcine sinus DAVFs. Yamaguchi et al. ${ }^{3}$ speculated that the DAVFs in the falx cerebri previously reported were drained via

\footnotetext{
${ }^{1}$ Department of Neurosurgery, Aizawa Hospital, Matsumoto, Nagano, Japan

${ }^{2}$ Department of Neurosurgery, Ina Central Hospital, Ina, Nagano, Japan
}

Received: July 8, 2020; Accepted: October 19, 2020

Corresponding author: Daisuke Satoh. Department of Neurosurgery, Aizawa Hospital, 2-5-1, Honjou, Matsumoto, Nagano 390-8510, Japan

Email: ds726@m7.dion.ne.jp

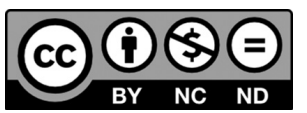

This work is licensed under a Creative Commons Attribution-NonCommercialNoDerivatives International License.

(C2021 The Japanese Society for Neuroendovascular Therapy falcine sinus and those cases must be falcine sinus DAVFs in their case report and literature review. However, the speculation has not been clarified. Here, we present a case of DAVFs in the falx cerebri, in which marked small vascular channels representing falcine venous plexus were visualized. Anatomy of falcine venous channels, falcine sinus, and their relationships with DAVFs are also discussed.

\section{Case Presentation}

A 59-year-old male was hospitalized with a slight traumatic subarachnoid hemorrhage caused by a head blow. He had a past history of head trauma by the traffic accident about 40 years ago. On admission, MRI T2-weighted image incidentally showed flow voids in the left occipital region and CTA revealed an asymptomatic DAVF in the falx cerebri (Fig. 1). The DAVF was supplied by the right middle meningeal artery (MMA), left superficial temporal artery (STA), bilateral occipital arteries (OAs) and bilateral posterior meningeal arteries, draining into the straight sinus through a dilated cortical vein on DSA (Fig. 2). There was no pial feeding artery from either of posterior cerebral arteries. Those multiple feeders converged to a venous pouch contiguous with radially shaped small venous channels in the falx cerebri. This venous configuration seemed to represent falcine venous plexus (Fig. 3).

Two months later, we performed a selective transarterial embolization because the reflux of shunt flow to the cortical 

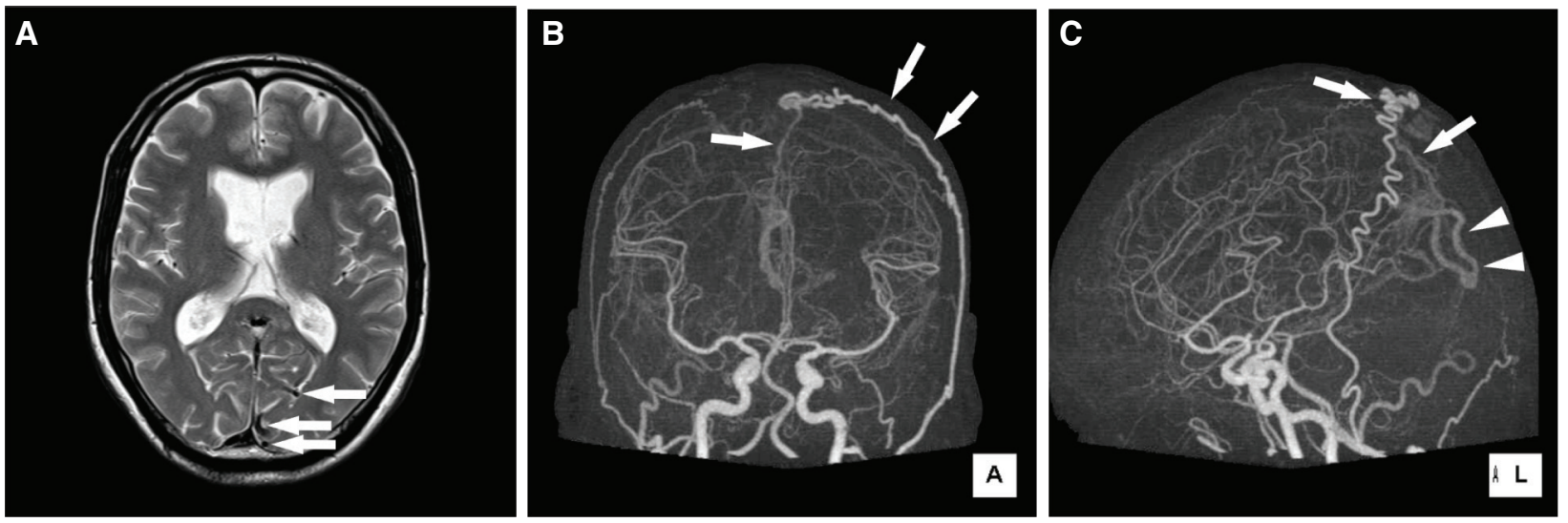

Fig. 1 (A) MRI T2-weighted image showing flow voids in the left occipital region (arrows). (B and C) CTA showing an occipital DAVF drained to the dilated cortical vein (arrowheads). CTA: computed tomography angiography; DAVF: dural arteriovenous fistula; STA: mainly fed by the left STA through the falcine artery (arrows) and superficial temporal artery
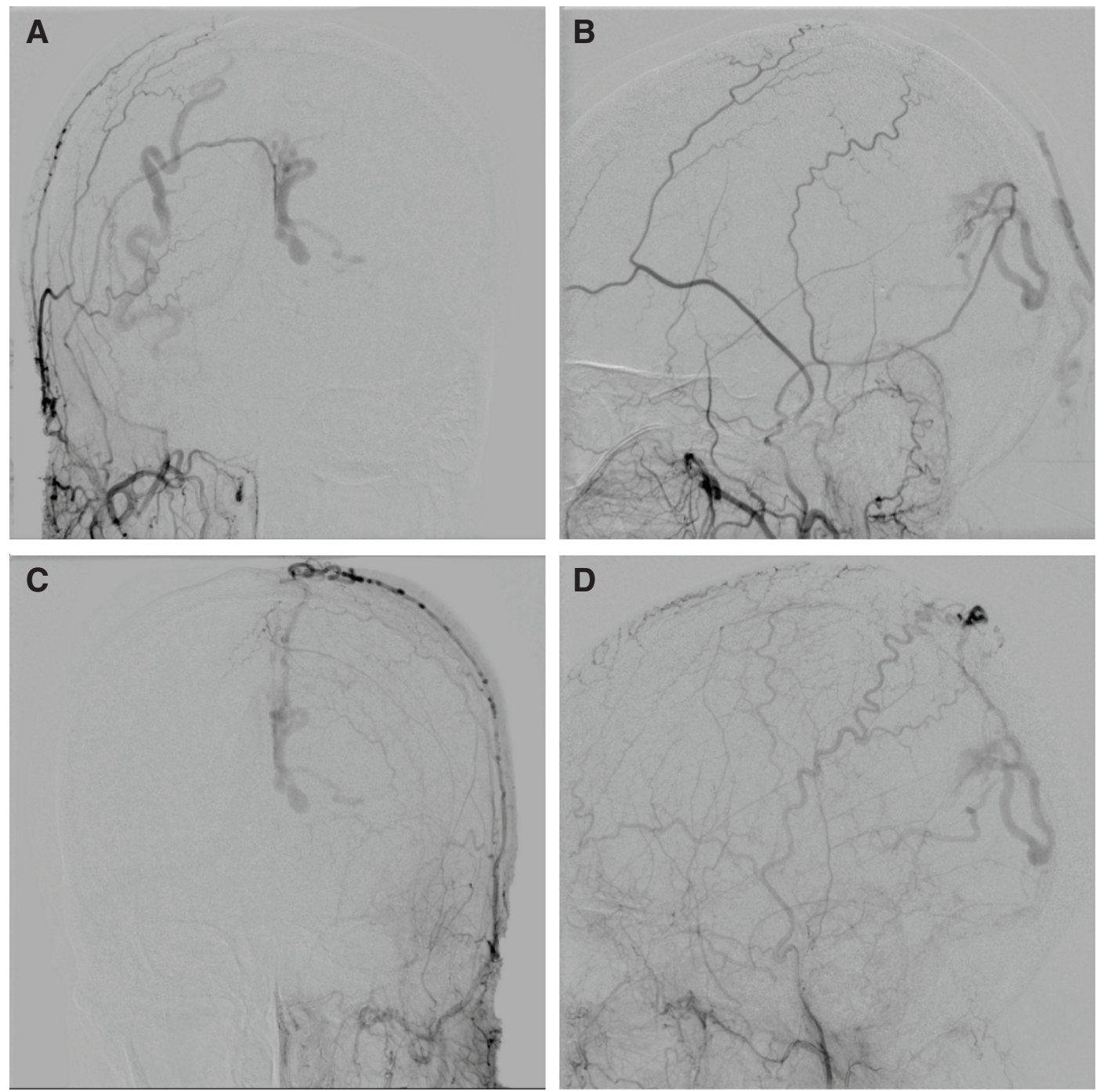

Fig. 2 (A and B) Right external carotid angiogram showing the DAVF fed by the right MMA. (C and $\mathbf{D}$ ) Left external carotid angiogram showing the DAVF fed by the left STA. DAVF: dural arteriovenous fistula; MMA: middle meningeal artery; STA: superficial temporal artery 

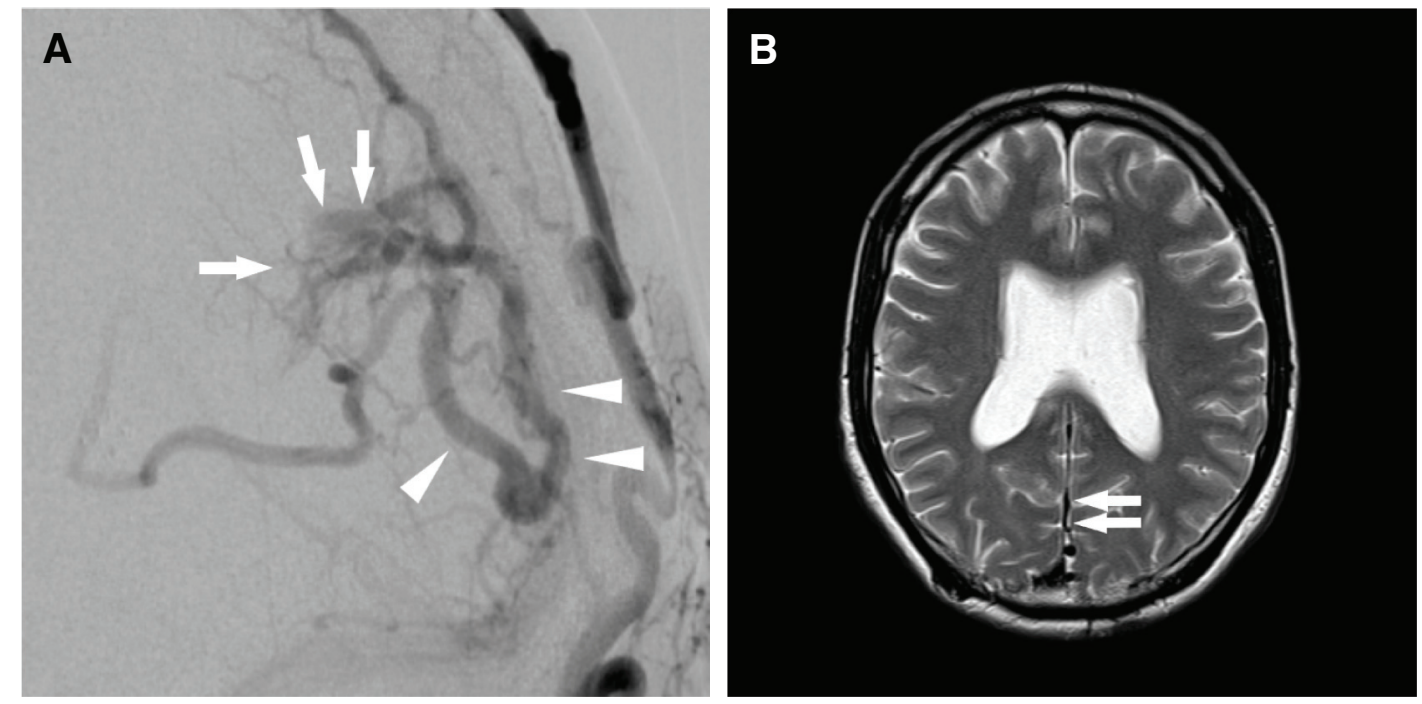

Fig. 3 (A) DSA showing shunt point of the DAVF located in the falx with prominent falcine venous plexus (arrows) and the DAVF drained into the dilated cortical vein (arrowheads). (B) MRI T2-weighted image showing the venous plexus located in the falx (arrows). DAVF: dural arteriovenous fistula

vein was evident. Under local anesthesia, a 7.2Fr introducer sheath was inserted into the right femoral artery. Following arterial access, $4000 \mathrm{U}$ of heparin was injected. First, a 7Fr Roadmaster STA 90cm (Goodman, Aichi, Japan) was guided to the left external carotid artery and a microcatheter (Marathon, Medtronic, Minneapolis, MN, USA) was navigated to the left STA over a 0.010 guidewire (CHIKAI-10, ASAHI INTECC, Aichi, Japan) through a 4Fr Cerulean G (Medikit, Tokyo, Japan) using as a distal access catheter (DAC) and guiding catheter. 20\% N-butyl 2-cyanoacrylate (NBCA) was injected through the microcatheter to reduce the shunt flow, then the 1t-STA was occluded in its proximal part. Next, the 7Fr Roadmaster was guided into the right external carotid artery and a Marathon microcatheter was advanced to distal segment of the right MMA through the same DAC and guiding catheter. After the test injection from the microcatheter, 20\% NBCA was slowly injected through the microcatheter into the drainer beyond the shunt point, where the glue cast spread in the venous plexus (Fig. 4). Right external carotid artery angiography showed a minimal shunt flow from the right OA remaining, so the right OA was occluded with particles (Avitene, Davol, Warwick, RI, USA). Postprocedural angiography revealed complete occlusion of the DAVF. Follow-up angiography 3 months after embolization showed no recurrence of DAVFs (Fig. 5). Postprocedural CT revealed the glue cast existing in the falx cerebri (Fig. 6). No complications associated with the procedure were evident.

\section{Discussion}

The DAVF in the falx cerebri is considered rare and only seven cases have been reported to our best knowledge. ${ }^{3-9}$ ) Most of their cases seem to associate with the falcine sinus. Our case revealed a shunted venous pouch connecting small venous channels in the falx. The falcine sinus was not seen. Those findings suggest the DAVF located in the vasculature of venous plexus in the falx cerebri. Tubbs et al. ${ }^{1)}$ reported the existence of venous plexuses in the falx cerebri in their cadaveric study. They found that all specimens had an extensive network of small venous tributaries within the falx that were concentrated primarily in its posterior one third. In that posterior segment, these structures were usually more pronounced in the inferior two-thirds. Tatarli et al. ${ }^{10)}$ evaluated the falcine venous plexus anatomically using scanning electron microscopy, and reported that the falcine venous plexus is a network of venous channels existing within the connective tissue of the falx; the sizes and patterns of communication of these structures showed regional differences, and in the posterior third of the falx cerebri the vessels of the falcine venous plexuses had the largest diameter. In our case, the falcine DAVF was located in its posterior one-third and inferior two-thirds, and in the segment, the falcine veins have large diameter, so the venous plexus might be confirmed angiographically.

As for the drainers of the DAVFs in the falx cerebri, Yamaguchi et al. ${ }^{3)}$ speculated that part of the drainers were the 

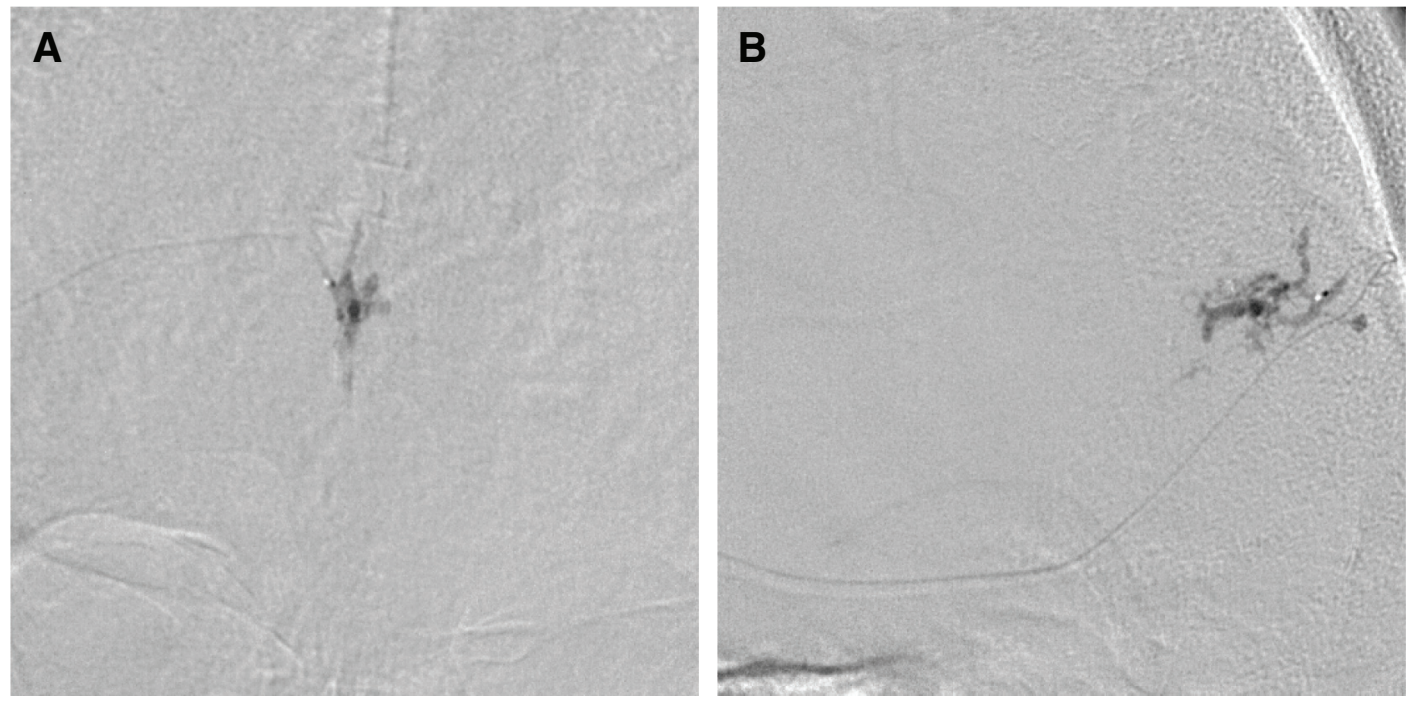

Fig. 4 Note that $20 \%$ NBCA was injected through the microcatheter to the drainer beyond the shunt point, and the glue cast spread in the venous plexus. (A) Anterior-posterior view and (B) lateral view. NBCA: N-butyl 2-cyanoacrylate
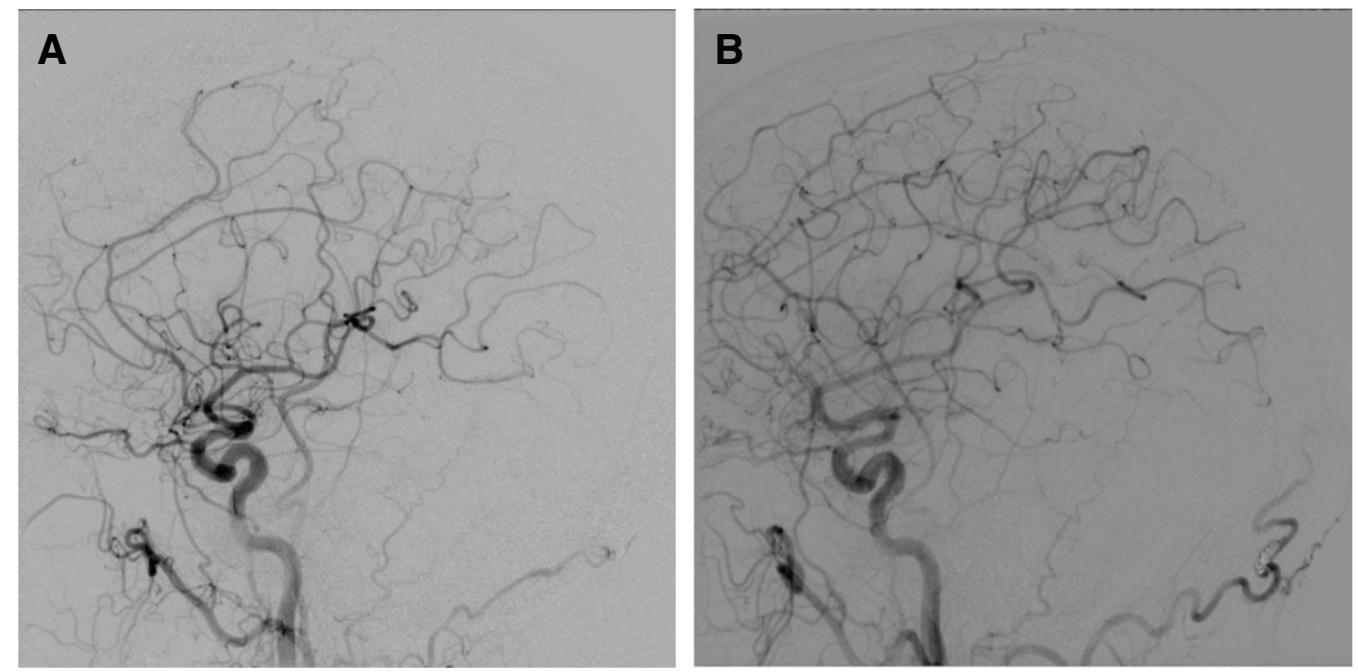

Fig. 5 Three months after procedural, DSA showing complete occlusion of DAVF. (A) Left common carotid artery injection and (B) right common carotid artery injection. DAVF: dural arteriovenous fistula

falcine sinuses draining into the SSS by their analysis. ${ }^{3}$ However, their speculation has not been confirmed. Tubbs et al. ${ }^{1)}$ reported that all falcine venous plexuses communicated with the ISS, and $63 \%$ communicated with the SSS, and they also introduced the comment by MG Yasargil that the sinus in the falx might receive a bridging vein. In our case, the DAVF draining into only the cortical vein through the bridging vein demonstrate communication between the bridging vein and the falcine venous plexus, and we consider that in a process of the formation of the DAVF in the present case, network channels between the venous plexus and SSS (or ISS) may have occluded, and as a result, only the reflux to the cortical vein remained. Lantz et al. ${ }^{11)}$ reported a similar case of probable falcine DAVF draining into only the cortical vein through the bridging vein. We suspect that their case was also the falcine DAVF through the falcine venous channels (plexus) without the connection to falcine sinus.

As to the treatment of the falcine venous plexus DAVF, transvenous embolization would not be feasible because of the difficulty in approaching and embolizing the venous plexus. We selected a transarterial embolization with NBCA in this case, while as recently reported for DAVFs, Onyx (Medtronic, Minneapolis, MN, USA) would be a useful alternative as well. ${ }^{12-14)}$ 

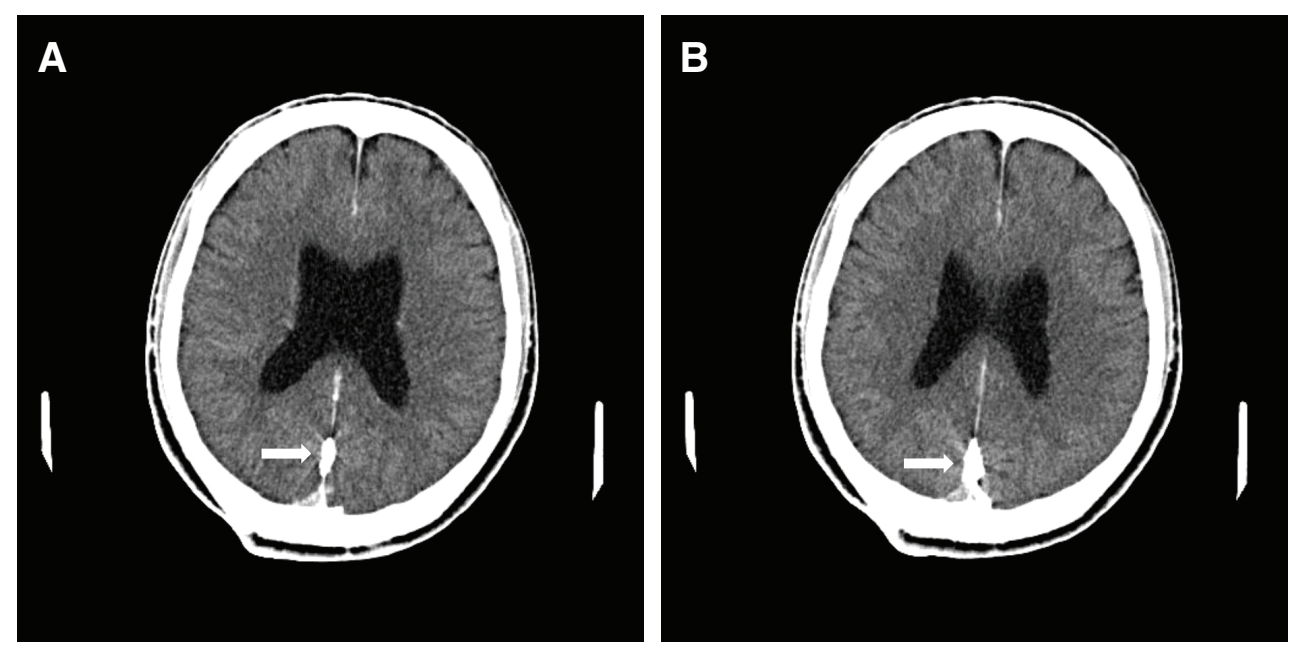

Fig. 6 (A and B) Postprocedural CT revealing the glue cast existing in the falx cerebri (arrow).

\section{Conclusion}

We experienced a rare case of DAVF in the flax with prominent falcine venous plexus that was successfully treated by a transarterial embolization. In our case, the DAVF draining into only the cortical vein through the bridging vein demonstrate communication between the bridging vein and the falcine venous plexus.

\section{Disclosure Statement}

There is no conflict of interest for the main author or coauthors.

\section{References}

1) Tubbs RS, Loukas M, Louis RG, et al: Anatomy of the falcine venous plexus. $J$ Neurosurg 2007; 107: 155-157.

2) Ryu CW: Persistent falcine sinus: is it really rare? AJNR Am J Neuroradiol 2010; 31: 367-369.

3) Yamaguchi $\mathrm{T}$, Higaki A, Yokota $\mathrm{H}$, et al: A case of dural arteriovenous fistula in the falx cerebri: case report and review of the literature. NMC Case Rep J 2016; 3: 67-70.

4) Yoshioka S, Moroi J, Kobayashi S, et al: A case of falcine sinus dural arteriovenous fistula. Neurosurgery 2013; 73: E554-556.

5) Kosnik EJ, Hunt WE, Miller CA: Dural arteriovenous malformations. J Neurosurg 1974; 40: 322-329.

6) Agawa M, Kohno T, Sogabe K: [Dural arteriovenous malformation in the falx with subarachnoid hemorrhage]. No Shinkei Geka 1991; 19: 841-845. (in Japanese)

7) Kothbauer K, Huber P: Dural arteriovenous fistula in the falx cerebri. Neuroradiology 1994; 36: 616-618.

8) Ratliff J, Voorhies RM: Arteriovenous fistula with associated aneurysms coexisting with dural arteriovenous malformation of the anterior inferior falx. Case report and review of the literature. J Neurosurg 1999; 91: 303-307.

9) Koyanagi M, Kobayashi T, Oda M, et al: Dural arteriovenous fistula in the falx cerebri treated with transarterial embolization using n-butyl cyanoacrylate. Surg Neurol Int 2016; 7: S1057-S1059.

10) Tatarli N, Ceylan D, Canaz H, et al: Falcine venous plexus within the falx cerebri: anatomical and scanning electron microscopic findings and clinical significance. Acta Neurochir (Wien) 2013; 155: 2183-2189; discussion 2189.

11) Lantz ER, Lavine SD, Festa JR, et al: Acute confusional syndrome from a dural arteriovenous fistula. Neurosurgery 2009; 65: E208-209; discussion E9.

12) Cognard C, Januel AC, Silva NA, et al: Endovascular treatment of intracranial dural arteriovenous fistulas with cortical venous drainage: new management using Onyx. AJNR Am J Neuroradiol 2008; 29: 235-241.

13) Chandra RV, Leslie-Mazwi TM, Mehta BP, et al: Transarterial onyx embolization of cranial dural arteriovenous fistulas: long-term follow-up. AJNR Am J Neuroradiol 2014; 35: 1793-1797.

14) Sadeh-Gonike U, Magand N, Armoiry X, et al: Transarterial onyx embolization of intracranial dural fistulas: a prospective cohort, systematic review, and meta-analysis. Neurosurgery 2018; 82: 854-863. 